ARTICLE

\title{
Nano-spectroscopy of excitons in atomically thin transition metal dichalcogenides
}

Shuai Zhang (10 1, Baichang Li (10 2, Xinzhong Chen (10 3,4, Francesco L. Ruta1,5, Yinming Shao', Aaron J. Sternbach (1) ${ }^{1}$, A. S. McLeod (1) ${ }^{1}$, Zhiyuan Sun (1) ${ }^{1}$, Lin Xiong ${ }^{1}$, S. L. Moore (1) ${ }^{1}$, Xinyi Xu ${ }^{2}$, Wenjing Wu ${ }^{6}$,

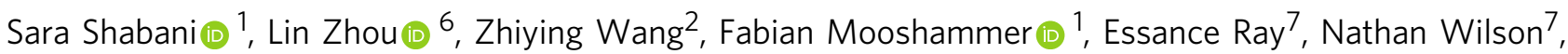
P. J. Schuck (1) 2, C. R. Dean (10 1, A. N. Pasupathy (1) ${ }^{1}$, Michal Lipson (1) ${ }^{8}$, Xiaodong Xu (1) 7, Xiaoyang Zhu (1) ${ }^{6}$, A. J. Millis ${ }^{1}$, Mengkun Liu (iD ${ }^{3,4}$, James C. Hone $\mathbb{D}^{2} \&$ D. N. Basov (i) ${ }^{1 \times}$

Excitons play a dominant role in the optoelectronic properties of atomically thin van der Waals ( $v d W$ ) semiconductors. These excitons are amenable to on-demand engineering with diverse control knobs, including dielectric screening, interlayer hybridization, and moiré potentials. However, external stimuli frequently yield heterogeneous excitonic responses at the nano- and meso-scales, making their spatial characterization with conventional diffraction-limited optics a formidable task. Here, we use a scattering-type scanning near-field optical microscope (s-SNOM) to acquire exciton spectra in atomically thin transition metal dichalcogenide microcrystals with previously unattainable $20 \mathrm{~nm}$ resolution. Our nano-optical data revealed material- and stacking-dependent exciton spectra of $\mathrm{MoSe}_{2}, \mathrm{WSe}_{2}$, and their heterostructures. Furthermore, we extracted the complex dielectric function of these prototypical vdW semiconductors. s-SNOM hyperspectral images uncovered how the dielectric screening modifies excitons at length scales as short as few nanometers. This work paves the way towards understanding and manipulation of excitons in atomically thin layers at the nanoscale.

\footnotetext{
${ }^{1}$ Department of Physics, Columbia University, New York, NY 10027, USA. ${ }^{2}$ Department of Mechanical Engineering, Columbia University, New York, NY 10027, USA. ${ }^{3}$ National Synchrotron Light Source II, Brookhaven National Laboratory, Upton, NY 11973, USA. ${ }^{4}$ Department of Physics and Astronomy, Stony Brook University, Stony Brook, NY 11794, USA. ${ }^{5}$ Department of Applied Physics and Applied Mathematics, Columbia University, New York, NY 10027, USA. ${ }^{6}$ Department of Chemistry, Columbia University, New York, NY 10027, USA. ${ }^{7}$ Department of Physics, University of Washington, Seattle, WA 98195, USA.

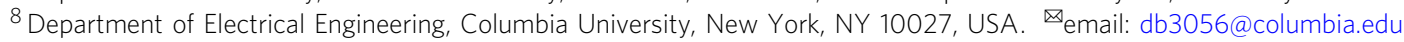


E xcitons, bound electron-hole pairs, govern the optical properties of two-dimensional transition metal dichalcogenides (TMDs) over near-infrared (IR) and visible frequencies due to their large binding energy and prominent oscillator strength ${ }^{1}$. Notably, all excitonic parameters in TMDs, including their resonance energies as well as both radiative and non-radiative lifetimes, can be manipulated on demand. Relevant control mechanisms include electric and magnetic fields, heterostructuring, moiré superlattice potentials ${ }^{2-5}$, local strain ${ }^{6}$, ultrafast optical excitation ${ }^{7,8}$, dielectric screening 9 , among others. The vast majority of experimental studies of excitonic phenomena in TMDs have been carried out by far-field optical methods with wavelength-limited spatial resolution. Nanoscale probes of the engineered excitonic electrodynamics are required to explore heterogeneous features. Nano-probe experiments reported here have the potential to map out the spatial evolution of exciton resonance energies in structures with (spatially) controlled dielectric screening and interlayer hybridization. In addition, nano-spectroscopy can document the potential role of extrinsic factors, such as defects and impurities, especially wrinkles and contaminations common in atomically layered heterostructures. Finally, applications of TMDs in nanophotonics and optoelectronics will benefit from characterization of excitons in nanodevices with sub-diffractional feature sizes that we demonstrated in our study.

A variety of scanning probe optical methods are in general suitable for the nanoscale characterization of the excitonic properties. Specifically, tip-enhanced photoluminescence (TEPL) studies have uncovered aspects of nanoscale excited state recombination in TMDs ${ }^{10-19}$. Complementary to the TEPL, absorption spectra encode not only the exciton resonance energy but also the oscillator strength and damping rate, offering additional detailed inquiry into the excitonic response. In principle, local absorption spectra acquired by scattering-type scanning near-field optical microscopy (s-SNOM) allow one to obtain the complex dielectric function $^{20}, \varepsilon(\omega)=\varepsilon_{1}(\omega)+\mathrm{i} \varepsilon_{2}(\omega)$ and make it possible to probe the engineered excitons at the nanoscale. This latter capability has been extensively used to study plasmons and phonons in other classes of van der Waals (vdW) materials ${ }^{21-23}$. However, formidable challenges so far precluded quantitative nano-spectroscopy of excitonic absorption at near-IR/visible (NIR/Vis) by means of s-SNOM ${ }^{24}$. For example, the reduced wavelength gives rise to more scattered light from the AFM tip shank, resulting in increased artificial background; the s-SNOM phase is more prone to optical path length drift as the wavelength is reduced.

Here, we coupled an ultra-stable and tunable continuous-wave Ti-sapphire laser ( $M$ squared) to a commercial s-SNOM (Neaspec $\mathrm{GmbH}$ ) and investigated the nanoscale excitonic spectra in monolayer TMD semiconductors and heterostructures. The materials of interest are monolayers of $\mathrm{WSe}_{2}$ and $\mathrm{MoSe}_{2}, \mathrm{MoSe}_{2} /$ $\mathrm{WSe}_{2}$ heterobilayers, as well as $\mathrm{WSe}_{2}$ trilayers. The exciton resonance energy, radiative lifetime, and the damping rate in vdW monolayers were extracted from the s-SNOM spectra with hitherto unattainable spatial resolution of $20 \mathrm{~nm}$. After documenting the ability of s-SNOM to probe excitons in atomically thin semiconductors with the nanoscale spatial resolution, we investigated heterostructures with lateral dimensions smaller than the diffraction limit of NIR/vis light. We show that intralayer excitons in $\mathrm{MoSe}_{2} / \mathrm{WSe}_{2}$ heterostructures red-shift relative to their counterparts in isolated monolayers due to the enhanced dielectric screening. The screening length is found to be shorter than the spatial resolution $(20 \mathrm{~nm})$ of our experiments and is attributed to the small radius of tightly bound excitons. The s-SNOM spectra were also applied to probe excitons altered by interlayer hybridization in a multi-layer $\mathrm{WSe}_{2}$ crystal.

\section{Results and discussion}

Experimental methods and samples preparation. The s-SNOM is based on a tapping mode atomic force microscope (AFM). A $\mathrm{Pt}-\mathrm{Ir}$ coated AFM tip is illuminated by a focused NIR/vis beam from a tunable continuous-wave laser, as shown in Fig. 1a. An intense optical field forms in the vicinity of the tip apex and interacts with the TMD crystals underneath it. The light backscattered from the tip is registered by a silicon detector and then demodulated at the high harmonics of the tip-tapping frequency via the pseudo-heterodyne interferometric scheme. This demodulation method allows one to isolate the genuine near-field signals from the undesired far-field background ${ }^{25,26}$. By scanning the tip on the sample, complex near-field signal is recorded with a resolution of $\sim 20 \mathrm{~nm}$ (Fig. 1b and Supplementary Fig. 1) and denoted as $s e^{\mathrm{i} \varphi}$, where $s$ and $\varphi$ are amplitude and phase, respectively. Atomically thin TMD monolayers and their heterostructures were prepared by mechanical exfoliation and stamped on a h-BN substrate. The spectral range used to investigate the samples is $1.50-1.75 \mathrm{eV}$, which covers the $1 \mathrm{~s}$ exciton resonance energies of $\mathrm{WSe}_{2}$ and $\mathrm{MoSe}_{2}$ monolayers (see inset in Fig. 1b). In this energy range, the near-field signal from h-BN is frequencyindependent ${ }^{27}$ and can be utilized as reference for spectroscopic data for TMD materials. The normalized near-field amplitude and phase at the $n$-th harmonic and energies $\omega$ are denoted by $s_{n}(\omega) / s_{n}(h-\mathrm{BN})$ and $\varphi_{n}(\omega)-\varphi_{n}(h-\mathrm{BN})$, respectively. A combination of our ultra-stable laser and scanner system allowed us to demodulate the scattering signal at the fifth harmonic of the tapping amplitude; high harmonic data is imperative for obtaining genuine near-field contrast devoid of far-field artifacts.

To display the excitonic responses in the near field, in Fig. 1c-e we show representative $s-S N O M$ images for a sample with monolayers of $\mathrm{MoSe}_{2}$ and $\mathrm{WSe}_{2}, \mathrm{MoSe}_{2} / \mathrm{WSe}_{2}$ heterobilayer, and unobscured h-BN all in the same field of view (FOV) of our apparatus. An AFM topographic image is shown in Fig. 1c, where a substantial height variation from left to right is prompted by a terrace in the underlying h-BN substrate. Nevertheless, regions of monolayer, bilayer, and multilayer TMDs can be readily identified by their topographic height. Our layer thickness assignment is further confirmed by the second harmonic generation (SHG) data (see Supplementary Figs. 2 and 3). The co-located images of topography (Fig. 1c), near-field scattering amplitude (Fig. 1d), and phase (Fig. 1e) are all acquired simultaneously. The amplitude image (Fig. 1d) reveals nearly uniform signals collected within terraces demarcated by boundaries between the terraces. We acquired images in Fig. 1d, e by setting the excitation photon energy at $1.68 \mathrm{eV}$. At this energy, a strong phase contrast between the $\mathrm{WSe}_{2}$ monolayer and h-BN substrate is observed (Fig. 1e). On the contrary, no discriminable phase contrast is found for the $\mathrm{MoSe}_{2}$ monolayer. The observation is readily understood as the near-field phase is related to the absorption ${ }^{28}$. The different excitonic absorption characteristics of the TMDs can give rise to phase contrast. According to our hyperspectral data in Fig. 2, the photon energy of $1.68 \mathrm{eV}$ is close to the exciton resonance in $\mathrm{WSe}_{2}$ monolayer and does not overlap with the excitonic band of $\mathrm{MoSe}_{2}$ centered at $1.58 \mathrm{eV}$. Our data reveal that the prominent nano-optical contrast in Fig. 1 is rooted in the local excitonic response of TMD monolayers. It should be noted that the contributions of thermal effect and charge effect to the near-field contrast can be ruled out by the high harmonic demodulation as also evident in the nearfield spectra shown in following sections.

Near-field nano-spectroscopy of excitons in $\mathrm{WSe}_{2}$ monolayer. For quantitative analysis of the $\mathrm{WSe}_{2}$ monolayer excitonic response, we carried out raster-scanned nano-imaging while 


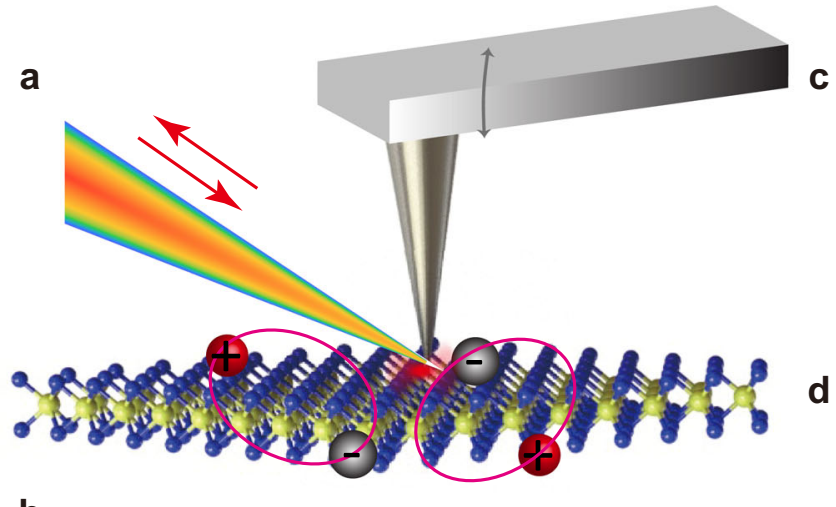

b
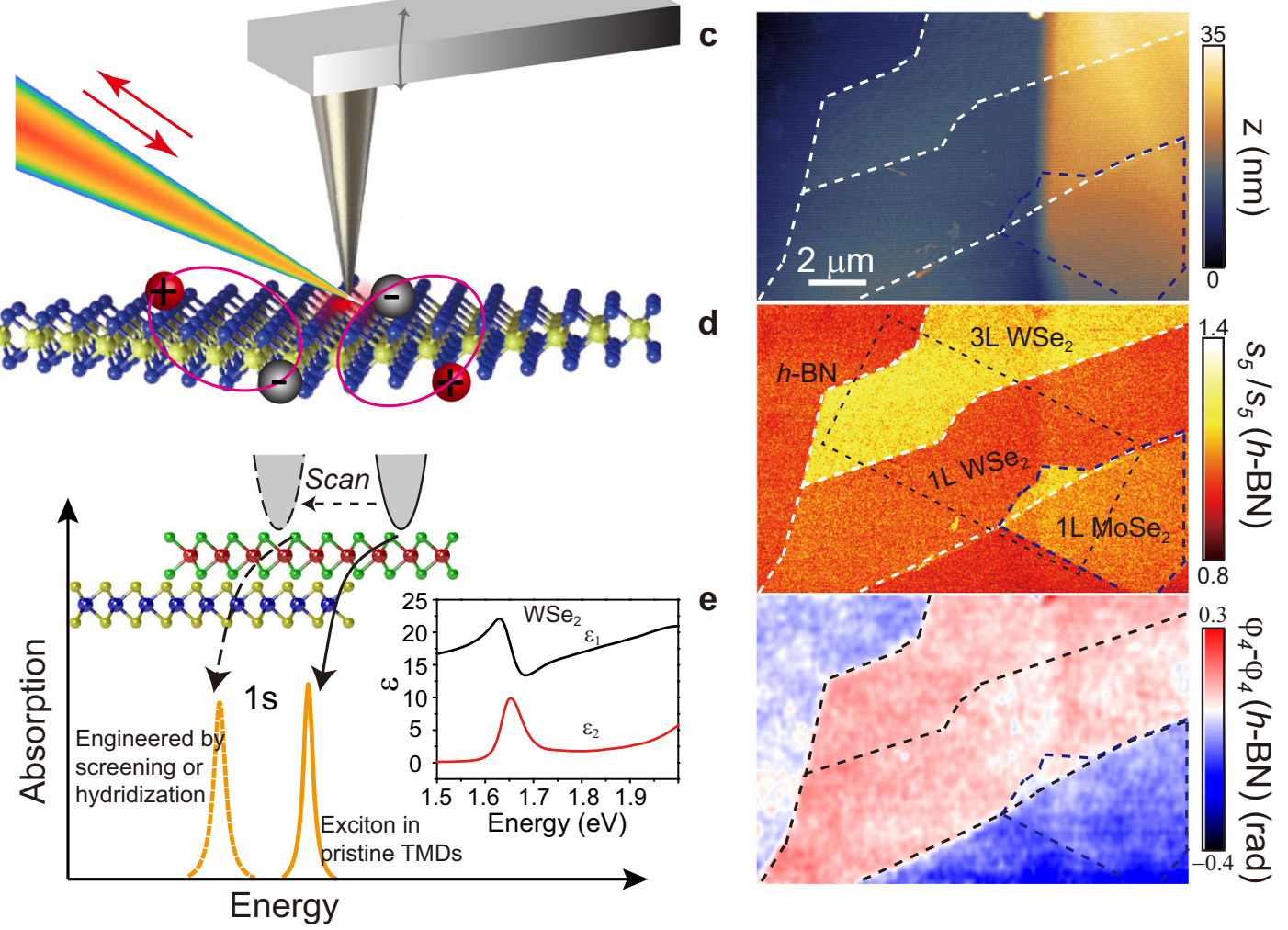

Fig. 1 Near-field nano-probing of excitons in atomically thin transition metal dichalcogendies (TMDs). a Schematic illustration of near-field measurement of excitons. The metallized AFM tip is illuminated by focused incident light and the tip-scattered light is collected. Pairs of electrons and holes with strong Coulomb interactions in atomically thin TMDs form excitons with large binding energies. The nanoscale response of excitons is extracted from the back-scattered light. $\mathbf{b}$ Scanning the tip across the TMD sample allows one to investigate the excitonic response below the diffraction limit and

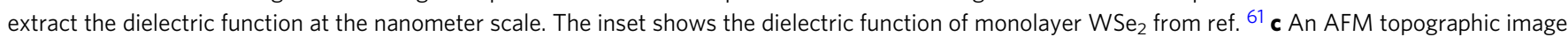
of the TMDs in this study. The height difference between the left and right regions is due to the h-BN terrace. d, e Near-field images of normalized scattering amplitude $s_{5}$ and phase $\varphi_{4}$ on the same region of (c). The excitation energy is $1.68 \mathrm{eV}$. The dashed bright and blue lines trace the edges of the different sample regions. The dashed rectangle marks the position of Fig. $2 \mathrm{a}$.

varying the photon energy with a step size of $\sim 10 \mathrm{meV}$. We have simultaneously collected topographic and near-field maps at each energy over the same FOV marked by the dashed rectangle in Fig. 1d. The topography image and height profile are shown in Supplementary Fig. 3. The unobscured h-BN in the upper left corner of Fig. 2 is used as a reference for normalization purposes. Representative maps of the normalized fifth-harmonic amplitude $s_{5}(\omega)$ and phase $\varphi_{5}(\omega)$ are shown in Fig. 2a, b, respectively. In these images, both the amplitude and phase signals in $\mathrm{WSe}_{2}$ monolayers vary systematically as a function of excitation energy. In particular, the phase images display a prominent contrast centered around $1.66 \mathrm{eV}$. Notably, we witness a highly uniform response within regions occupied by each of the TMD materials in Fig. 2. All the boundaries and some terraces are in a deeply sub-diffraction regime. Nevertheless, these features can be clearly discriminated in our s-SNOM images. To quantify the excitonic response, we start our analysis by averaging the near-field signals over the entire region occupied by $\mathrm{WSe}_{2}$ monolayer at each probing frequency. The averaging process will facilitate a more faithful determination of dielectrics function, which will be discussed later. The net result is that each data point in spectra plotted in Fig. 2c, d is representative of the entire region imaged at the corresponding energy in Fig. 2a, b. The spectra derived from images show prominent resonance characteristics: the amplitude signal reveals a dispersive behavior around $1.66 \mathrm{eV}$, whereas the phase signal exhibits a peak centered at the same energy.
We modeled experimental near-field spectra of $\mathrm{WSe}_{2}$ in Fig. 2 with the Lorentzian form of the dielectric function ${ }^{29-31}$ :

$$
\varepsilon(\omega)=\varepsilon_{\infty}-\frac{c}{\omega_{0} d} \frac{\gamma_{r, 0}}{\omega-\omega_{0}+i\left(\frac{\gamma_{n r}}{2}+\gamma_{d}\right)}
$$

where $\varepsilon_{\infty}$ is the high-frequency permittivity limit and originates from all transitions with frequencies beyond the spectral range investigated here, $c$ is the speed of light in vacuum, $\omega_{0}$ is the exciton resonance energy, $d$ is the monolayer thickness, $\gamma_{r, 0}, \gamma_{n r}$, $\gamma_{d}$ are radiative, non-radiative, and dephasing decay rates, respectively. The oscillator strength of the Lorentzian in Eq. 1 is related to the radiative rate $\gamma_{r, 0}$. We choose the point dipole model $^{32}$ to interpret the data as it is well documented to capture the response of atomically thin samples laid on thick substrates $^{32,33}$. As shown in Fig. 2c, d, the point dipole model with Eq. 1 as the input dielectric function provides an adequate fit to the data (see SOM for details on fitting).

Now we analyze the parameters inferred from the fitting procedure (Table 1). The obtained dielectric function is plotted in Fig. 2e. The radiative rate for monolayer $\mathrm{WSe}_{2}$ is $1.4 \mathrm{meV}$, which corresponds to the radiative lifetime $\tau_{0}=\frac{\hbar}{2 \gamma_{r .0}}$ of $\sim 233 \mathrm{fs}$. This latter finding is consistent with the first principles theoretical values $^{34}$ and experimental results derived from the ultrafast exciton $1 \mathrm{~s}-2 \mathrm{p}$ transition, which reveal $150 \mathrm{fs}$ radiative lifetime in $\mathrm{WSe}_{2}$ monolayers ${ }^{35}$. The shorter radiative lifetime compared to that of conventional semiconductors stems from the exceptionally 


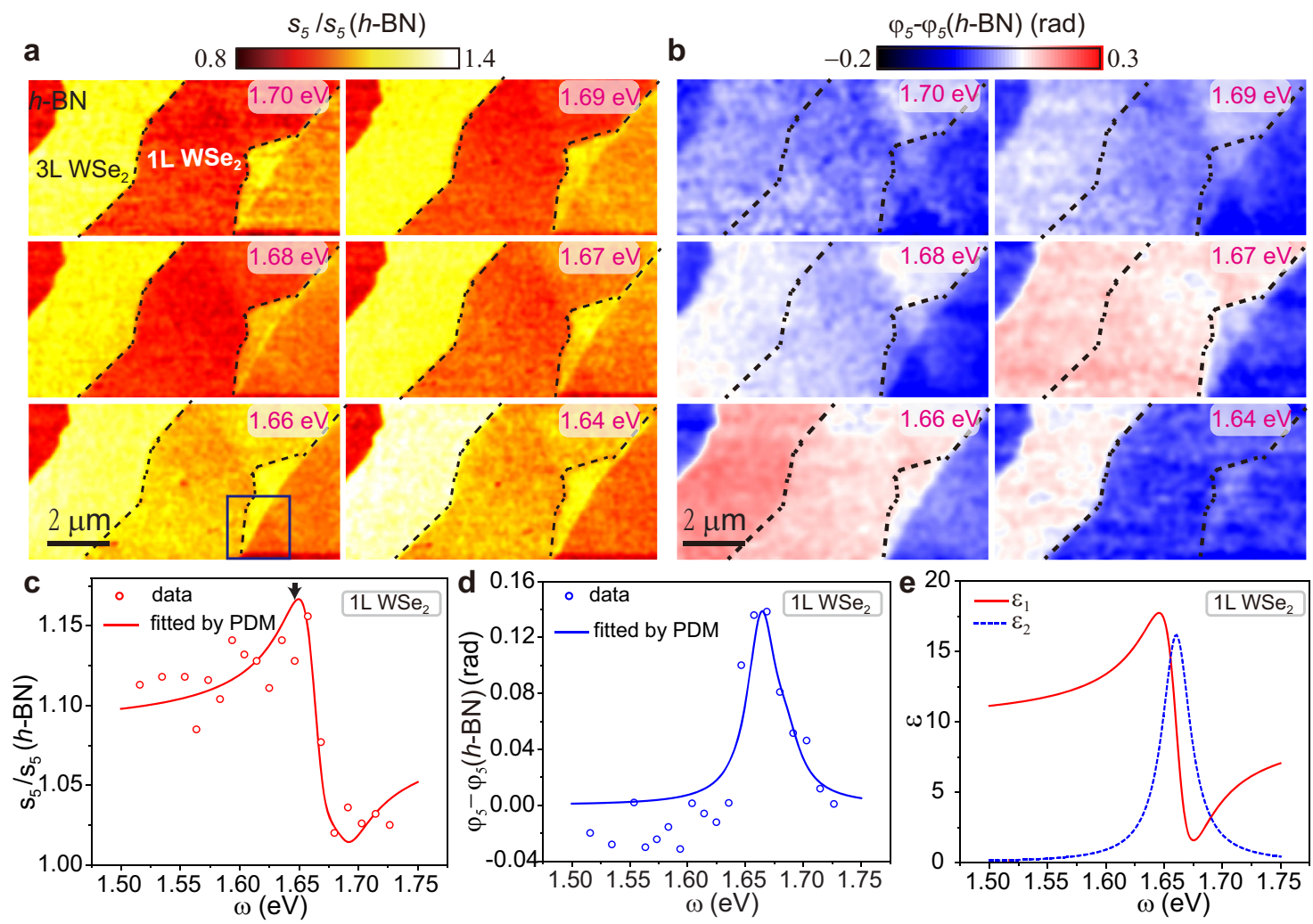

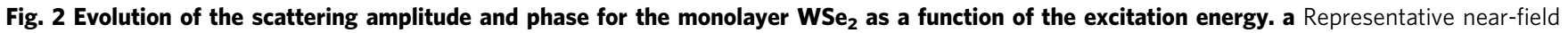
images of the normalized scattering amplitude $s_{5}(\omega) / s_{5}(h-B N)$. The excitation energies are indicated in the images, which are acquired by scanning over a rectangular area marked with the dashed line in Fig. 1d. Boundaries of the monolayer $\mathrm{WSe}_{2}$ region are displayed with black dashed lines. The area enclosed by blue lines in bottom left image was investigated in Fig. 4 a. $\mathbf{b}$ Near-field images of the normalized phase $\varphi_{5}(\omega)-\varphi_{5}(h-B N)$. The images with the same excitation energy in $\mathbf{a}$ and $\mathbf{b}$ are acquired simultaneously. An analogous dataset demodulated at the fourth harmonic is shown in Supplementary Fig. 5. c, $\mathbf{d}$ Normalized near-field amplitude $s_{5}(\omega) / s_{5}(h-\mathrm{BN})$ and phase $\varphi_{5}(\omega)-\varphi_{5}(h-\mathrm{BN})$ spectra for WSe $\mathrm{e}_{2}$ monolayer (data points). Fits to the spectra using the point dipole model (PDM) and a single Lorentz oscillator (Eq. 1) are shown as solid curves. The arrow in $\mathbf{c}$ marks the peak energy of PL spectra (Supplementary Fig. 4). e Dielectric function of $\mathrm{WSe}_{2}$ monolayer extracted from the fits with PDM in (c, d).

Table 1 The parameters of Lorentz model used to describe TMD dielectric function as obtained from fitting s-SNOM spectra with the point dipole model.

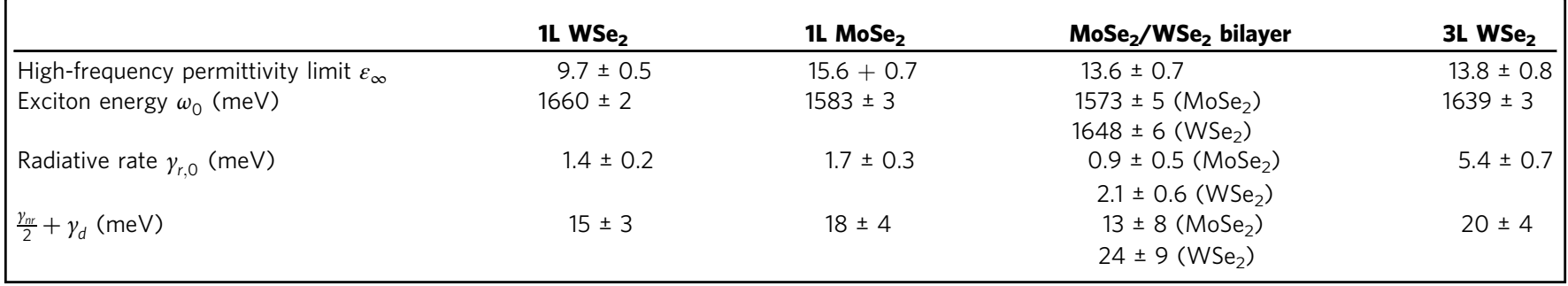

large exciton oscillator strength in two-dimensional TMDs, evidenced by the high optical absorption in monolayer ${ }^{36,37}$. We obtained the damping rate $\frac{\gamma_{n r}}{2}+\gamma_{d}=15 \mathrm{meV}$. We remark that this substantial damping at room temperature inhibits the real dielectric function from crossing zero, and thus the exciton polariton regime cannot be reached. Recently, reflection measurements at cryogenic temperature demonstrated the negative real dielectric function in the vicinity of the excitonic line ${ }^{38}$. Therefore, by reducing the damping rate, for example, through experiments conducted at cryogenic temperature ${ }^{31,38}$, it may become possible to access the exciton polaritons in monolayer TMDs. So far, all experiments on exciton polaritons in TMDs conducted at ambient required integration of the active semiconductor in various forms of photonic cavities ${ }^{39-41}$. A notable exception is an observation of the excited state Rydberg exciton polaritons demonstrated in pump-probe experiments for multilayer $\mathrm{WSe}_{2}$ at room temperature ${ }^{8}$.

Next, we comment on the contributions of bright and dark excitons to the s-SNOM spectra. These two types of excitonic species result from the spin splitting of the conduction band edge and they obey different optical selection rules ${ }^{42,43}$. In TMD literature, excitons that can be excited by light with polarization in the sample plane, as in normal incidence experiments, are referred to as bright excitons. Excitons that can be activated only 
when light polarization is perpendicular to the sample plane, are referred to as dark excitons. Dark excitons can be visualized using TEPL for TMD samples on metallic substrates because the electric field strength perpendicular to the sample surface is drastically enhanced $^{12,19}$. In contrast, the s-SNOM signal for atomically thin TMDs is governed by the in-plane dielectric function ${ }^{32,44}$, which arises from bright excitons (see SOM). To experimentally confirm the dominant role of bright excitons in our data, we compared the bright exciton resonance energy obtained from far-field PL spectra on the same sample ${ }^{45}$ with the resonance energy in our s-SNOM spectra. The bright exciton resonance energy from PL (see Supplementary Fig. 4) is about $1.66 \mathrm{eV}$, in close agreement with s-SNOM data. We, therefore, conclude that bright excitons dominate the s-SNOM response of monolayer and few-layer TMD crystals.

As discussed above, the s-SNOM spectra are determined by the dielectric function of the materials; whereas TEPL is determined by both the exciton population and the transition rate 46 and therefore is sensitive to sample parameters. Stain, defects, and contaminants in samples, particularly chemical vapor deposition (CVD) grown ones commonly used in TEPL experiments, can impact the exciton distribution and resonance energies and thus give raise to inhomogeneity of the TEPL. Here, to probe the intrinsic properties of TMDs, the samples are exfoliated from the high quality crystals grown by the self-flux method ${ }^{47}$ and fabricated by the contamination-free stacking method (details in "Methods"). The resulting high-quality sample possesses nearly uniform dielectric function, thereby exhibiting a nearly homogenous s-SNOM signal.

Excitonic spectra of TMD monolayers. Before exploring the nanoscale response of heterostructures and multilayers, we take a closer look at the near-field response of the constituent monolayers. The spectra of the normalized amplitude and phase for $\mathrm{MoSe}_{2}$ are shown in the middle panels of Fig. 3a, b (see Supplementary Fig. 6 for complete data sets). Each data point in these spectra is attained by averaging the near-field signal over the entire $\mathrm{MoSe}_{2}$ regions. The near-field spectra for $\mathrm{MoSe}_{2}$ are akin to those of $\mathrm{WSe}_{2}$ and exhibit nearly the same resonance features, manifesting as a derivative-type lineform of the amplitude and a peak in the phase. The resonance energy observed in $\mathrm{MoSe}_{2}$ using s-SNOM $(1.583 \mathrm{eV})$ is consistent with the dominant peak in PL spectra $^{48}$ (see Supplementary Fig. 4), supporting the notion of bright excitons. The radiative rate obtained by fitting the spectra for $\mathrm{MoSe}_{2}$ is as high as $1.7 \mathrm{meV}$ and is slightly larger than that of $\mathrm{WSe}_{2}$ (see Table 1). In general, the prominent radiative rates stem from the two dimensional nature of excitons in TMDs, which are tightly bound to monolayers and have a small exciton radius ${ }^{49}$. Even though the spectral lineforms of excitonic lines in $\mathrm{MoSe}_{2}$ and $\mathrm{WSe}_{2}$ are nearly identical, the near-field amplitude detected for $\mathrm{MoSe}_{2}$ is considerably larger than that of $\mathrm{WSe}_{2}$ below the exciton resonance energies (top and middle panels of Fig. 3a). We also found that the magnitude of the real part of the dielectric function $\varepsilon_{1}$ at energies below the exciton resonance is enhanced in $\mathrm{MoSe}_{2}$ compared to that of $\mathrm{WSe}_{2}$. This latter finding is consistent with the enhanced radiative rate in $\mathrm{MoSe}_{2}$.

$\mathrm{MoSe}_{2} / \mathrm{WSe}_{2}$ heterobilayers and WSe $\mathrm{W}_{2}$ trilayers. Now we turn to the spectra acquired for the $\mathrm{MoSe}_{2} / \mathrm{WSe}_{2}$ heterostructures with the width of less than $1 \mu \mathrm{m}$, as shown in the $s_{5}$ amplitude map (Fig. 3c, where HB denotes the heterobilayer region). The amplitude and phase spectra are displayed in the bottom panels in Fig. 3a, b, respectively. In these spectra, two resonances emerge at the energies close to the frequency positions of the exitonic bands in $\mathrm{MoSe}_{2}$ and $\mathrm{WSe}_{2}$ monolayers in Fig. 2 and Supplementary Fig. 6, indicating a dominant contribution of intralayer bright excitons. The role of interlayer excitons remains negligible in the data plotted in Fig. 3. Indeed, the resonance energies of indirect excitons $s^{4,50,51}(\sim 1.3-1.4 \mathrm{eV})$ fall outside the spectral range investigated in Fig. 3.

We analyzed spectra of the $\mathrm{MoSe}_{2} / \mathrm{WSe}_{2}$ heterostructure assuming that the dielectric function is given by the sum of two Lorentzians in Eq. 1. The fit with PDM shows that both the intralayer exciton resonance energies red-shift compared to their counterparts in monolayers (vertical dashed lines in Fig. 3a, b) Red-shift of the exciton resonance energies can also be clearly identified in the hyperspectral measurements of the amplitude $s_{5}$ and phase $\varphi_{5}$ (in Fig. 3d, e, respectively) along the line trace in Fig. 3c. The frequency red-shift of the two exciton resonances is around $10 \mathrm{meV}$ compared to their monolayer counterparts (Table 1 and Fig. 3a, b).

To unravel the origin of the red-shifts, the twist angle of the two layers comprising our heterostructure was characterized by polarization-resolved SHG experiments. The SHG data show that the twist angle between layers is either $15^{\circ}$ or alternatively $45^{\circ}$ (see Supplementary Fig. 2). Either of the two layer arrangements implies the misalignment between the Brillouin zones of monolayers ${ }^{51,52}$ and therefore produces only negligible hybridization between the two atomic planes. However, excitons in the heterostructure experience stronger dielectric screening compared to the isolated monolayers, because the dielectric function of TMDs is larger than that of h-BN $(\sim 4)$. Therefore, we conclude that in heterostructures with a large twist angle, the redshift of intralayer exciton resonance energies mainly arises from dielectric self-screening 9,52 .

Although the effect of dielectric screening on excitons has been widely investigated, the screening length is difficult to infer from previous reports based on diffraction-limited far-field data ${ }^{9,53}$. Here the screening length refers to the spatial span over which the exciton energies are altered by abrupt changes in the dielectric environment. s-SNOM experiments visualize the spatial evolution of the exciton lines across interfaces and thus can potentially quantify the screening length. Representative s-SNOM images in Fig. $4 \mathrm{a}, \mathrm{b}$ show that the amplitude and phase contrasts abruptly change across the boundary between the monolayers and heterostructure. From the high-resolution images and the line profiles (Fig. 4c), one can see that the exciton resonance energy is altered over the length scale of about $20 \mathrm{~nm}$, which is the spatial resolution in our experiment. We conclude that our nano-NIR/vis scans yield only the upper bound estimate of this length scale limited by the spatial resolution of our method. Our estimate is consistent with the theoretically predicted value of about $10 \mathrm{~nm}$ (ref. ${ }^{9}$ ). Figure $4 \mathrm{a}-\mathrm{c}$ displays the representative results affirming the s-SNOM contrast changes on the length scale of $\sim 20 \mathrm{~nm}$. Data in Figs. 2 and 3 uncovers the abruptness of these changes both in amplitude and phase signals over the entire frequency range. The totality of the data in Figs. 2-4 attest to the nanoscale sensitivity of our approach to the dielectric function.

In addition to the dielectric screening, interlayer hybridization also plays a role in controlling the excitonic properties of vdW materials, particularly in homostructures. Our nano-NIR/vis data provides an experimental access to interlayer hybridization in trilayer $\mathrm{WSe}_{2}$ exfoliated from $2 \mathrm{H}$-stacked crystal. We anticipate that in these crystals neighboring layers are twisted by an angle of $60^{\circ}$. The amplitude and phase spectra acquired on the $\mathrm{WSe}_{2}$ trilayer are shown in Fig. 4d, e, respectively, with fitting results presented in Table 1 . These data show that the exciton resonance energy decreases from $1.660 \mathrm{eV}$ in $\mathrm{WSe}_{2}$ monolayer (dashed vertical line in Fig. 4e) to $1.639 \mathrm{eV}$ in the trilayer (solid vertical 

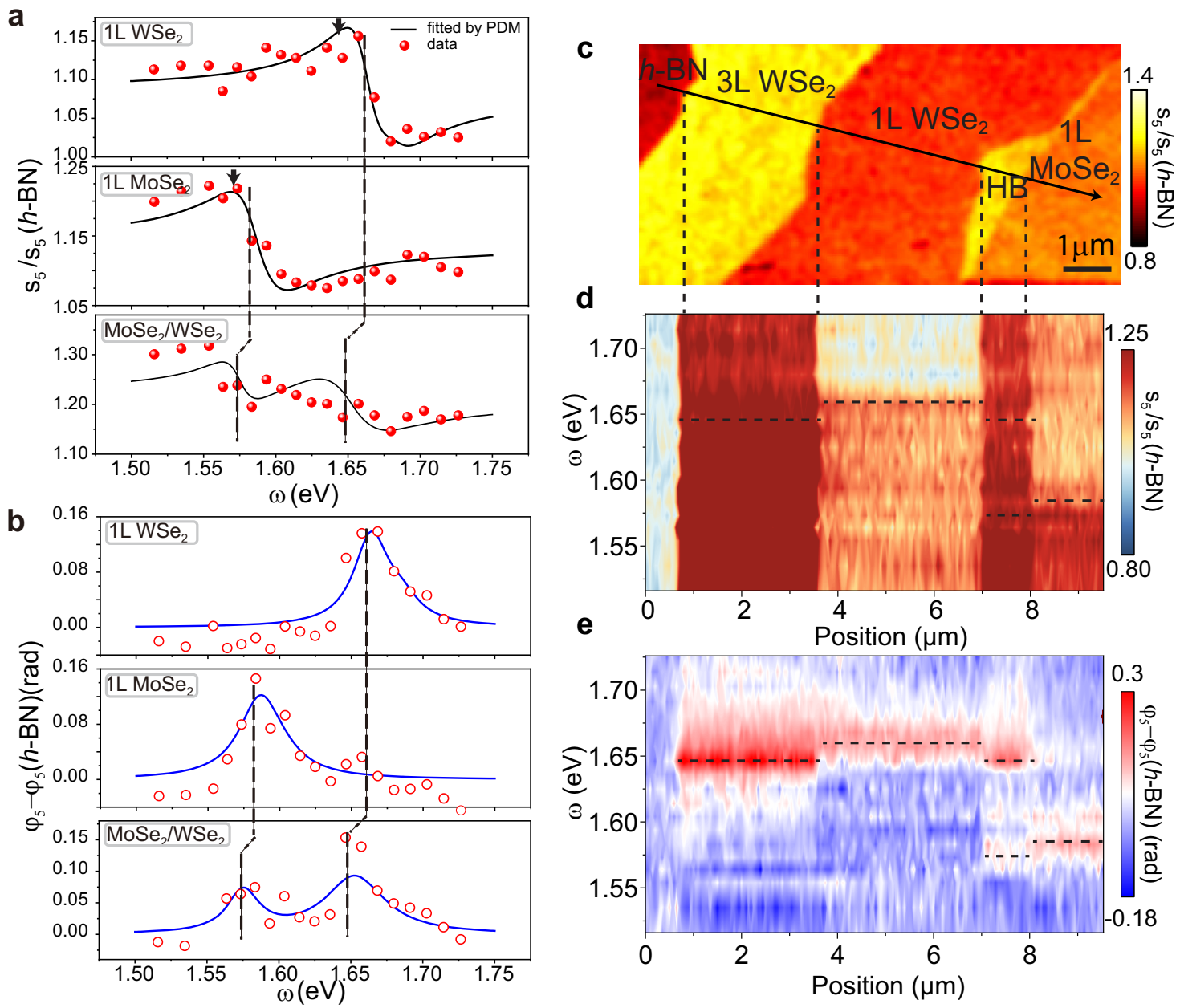

Fig. 3 Nano-spectroscopy of different types of excitons. a, b Normalized amplitude $s_{5}(\omega) / s_{5}(h-B N)$ and phase $\varphi_{5}(\omega)-\varphi_{5}(h-B N)$ spectra for different sample regions (data points). The near-field data is fitted by the point dipole model and Lorentz oscillators (solid curves). The vertical dashed lines are used to mark the exciton energies of $\mathrm{WSe}_{2}$ and $\mathrm{MoSe}_{2}$ extracted from the point dipole model. The arrows in a mark peak energies of the PL spectra (Supplementary Fig. 4). c Near-field image of the amplitude $s_{5}$ taken at an excitation energy of $1.52 \mathrm{eV}$. HB: heterobilayer. d, e Near-field amplitude $s_{5}$ and phase $\varphi_{5}$ evolution along the line trace shown in (c). The horizontal dashed lines in $\mathbf{d}$ and $\mathbf{e}$ denote the exciton energies extracted from the point dipole model.

lines in Fig. 4e). The indirect transitions in few-layer TMD crystals have negligible oscillator strength, and thus make only a minute contribution to the dielectric function and/or to the s-SNOM signal. Therefore, the observed redshift of the exciton resonance energy originates from direct transitions. For trilayer $\mathrm{WSe}_{2}$ with a twist angle of $60^{\circ}$, interlayer electronic hybridization splits both conduction and valence band edges at the corner of the Brillouin zone, thus reducing the energy gap and the corresponding exciton resonance energy $52,54,55$. Therefore, the large shift observed in $\mathrm{WSe}_{2}$ trilayer $(21 \mathrm{meV})$ is likely to be caused by the interlayer hybridization. We note that the dielectric screening could also reduce the exciton resonance energy, but its effect is subtle $(\sim 10 \mathrm{meV}$, as demonstrated in the last section), compared to the interlayer hybridization.

In summary, the bright excitonic responses of TMD monolayers were revealed by s-SNOM hyperspectral nano-imaging. New data allowed us to extract the exciton resonance energy, oscillator strength, and damping rate all with the nanometer resolution. Nano-NIR/vis spectra and images uncovered the spatial evolution of the dielectric screening and interlayer hybridization. We obtained the upper bound of the dielectric screening length of $20 \mathrm{~nm}$ limited by the achieved spatial resolution of s-SNOM in this work. Our results lay the groundwork for future spatio-temporal ${ }^{35,53}$ studies of excitonic states, including moiré excitons ${ }^{2,56-58}$, exciton liquid ${ }^{59}$, and exciton phase transitions ${ }^{60}$ in a wide range of quantum materials.

\section{Methods}

Sample preparation. Monolayers of $\mathrm{WSe}_{2}, \mathrm{MoSe}_{2}$, and $30 \mathrm{~nm}$ thick $\mathrm{BN}$ were exfoliated onto $\mathrm{SiO}_{2} / \mathrm{Si}$ using the standard scotch tape method. To prepare the heterostructure sample, a polydimethylsiloxane (PDMS) stamp was coated with thin polypropylene carbonate (PPC) film and subsequently attached onto a glass slide. Inverted stacking was performed on a dry transfer station with a rotatable heating stage. Thick BN and monolayers of $\mathrm{WSe}_{2}$ and $\mathrm{MoSe}_{2}$ were picked up subsequently, with controlled alignment. Finally, the heterostructure with PPC was peeled off from the PDMS and transferred to a clean $\mathrm{SiO}_{2} / \mathrm{Si}$ substrate at $120^{\circ} \mathrm{C}$. The twist angle of the heterostructure is determined by the SHG spectra from individual layers and their overlap region.

Near-field measurement. The nano-imaging was performed using a commercial s-SNOM (www.neaspec.com) based on a tapping-mode AFM. The tapping frequency and amplitude of the AFM are about $75 \mathrm{kHz}$ and $50 \mathrm{~nm}$, respectively. The light source is a tunable continuous laser from M Squared, including single frequency CW-532 nm module (EQUINOX), continuous-wave Ti-sapphire model with output range 700-1000 $\mathrm{nm}$ (SolsTiS), and frequency mixing module with tunable output range 1100-2200 nm (DFG-532). By focusing the laser beam onto the metallized AFM tip, we were able to probe the optical properties with 

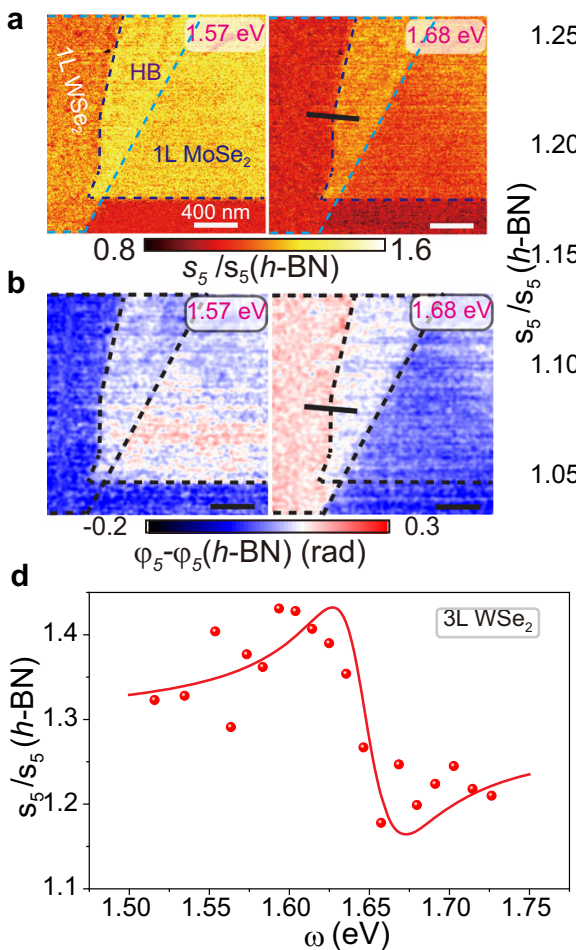
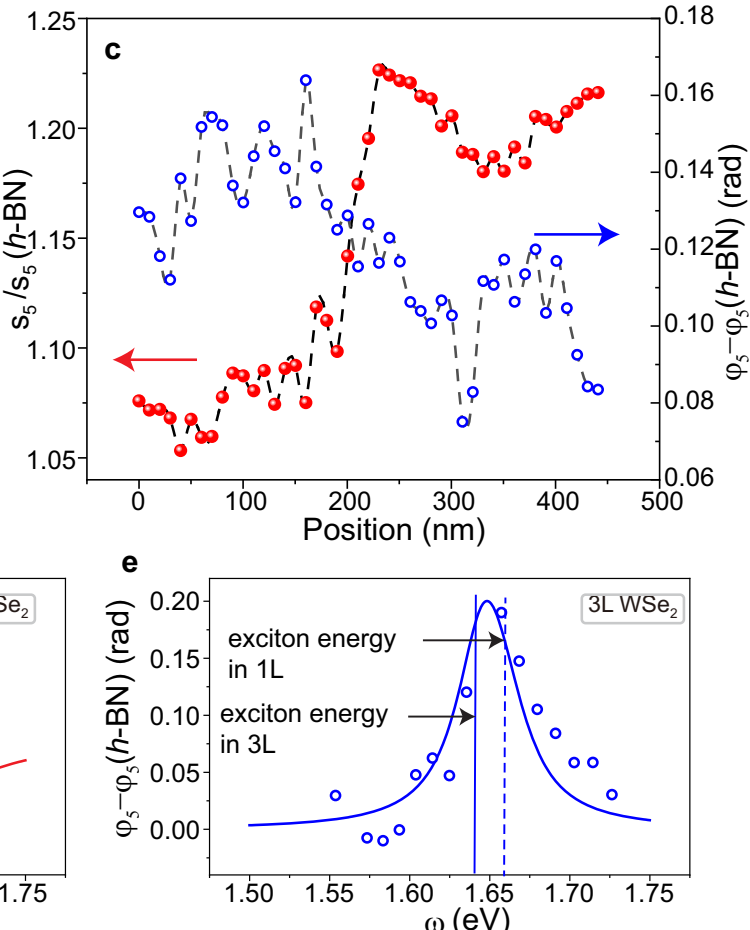

Fig. 4 Screening length of excitons in heterostructure and the effect of hybridization on the exciton resonance in WSe $\mathbf{e}_{\mathbf{2}}$ trilayer. $\mathbf{a}$, $\mathbf{b}$ Near-field images of the normalized amplitude $s_{5}(\omega) / s_{5}(h-\mathrm{BN})$ and phase $\varphi_{5}(\omega)-\varphi_{5}(h-\mathrm{BN})$. The images in the left and right panels were taken at $1.68 \mathrm{eV}$ (around the monolayer $\mathrm{WSe}_{2}$ exciton resonance energy) and $1.57 \mathrm{eV}$ (around monolayer $\mathrm{MoSe}_{2}$ exciton resonance energy), respectively. The boundaries of the materials are displayed with dash lines. The scale bars indicate $400 \mathrm{~nm}$. $\mathbf{c}$ Line profiles of the normalized amplitude and phase across the monolayer and heterobilayer along the black lines in the right panels in (a and b). d, e Normalized scattering amplitude $s_{5}(\omega) / s_{5}(h-B N)$ and phase $\varphi_{5}(\omega)$ spectra for WSe $e_{2}$ trilayer (data points). The near-field data are fitted by the point dipole model (solid curves). The vertical blue dashed and solid lines in e are used to mark the exciton energies of $\mathrm{WSe}_{2}$ monolayer and $\mathrm{WSe}_{2}$ trilayer extracted from the point dipole model, respectively.

subwavelength resolution. To this end, the back-scattered light is registered by pseudo-heterodyne interferometric detection and then demodulated at the $n$-th harmonics of the tapping frequency yielding background-free images. To eliminate the background, we chose $n=4$ and 5 in this work. In addition to the background elimination, the high harmonic signal can also reduce the detection depth, which is essential to detect the atomically thin TMDs.

\section{Data availability}

Raw files containing the unprocessed near-field images are available from the corresponding author upon reasonable request.

\section{Code availability}

Code used to fit the near-field spectra is available from the corresponding author upon reasonable request. This code is based on the NearFieldOptics python package, downloaded from a github repository from Alexander S. Mcleod (https://github.com/ asmcleod/NearFieldOptics).

Received: 2 August 2021; Accepted: 6 January 2022;

Published online: 27 January 2022

\section{References}

1. Wang, G. et al. Colloquium: Excitons in atomically thin transition metal dichalcogenides. Rev. Mod. Phys. 90, 021001 (2018).

2. Yu, H., Liu, G.-B., Tang, J., Xu, X. \& Yao, W. Moiré excitons: From programmable quantum emitter arrays to spin-orbit-coupled artificial lattices. Sci. Adv. 3, e1701696 (2017).

3. Tran, K. et al. Evidence for moiré excitons in van der Waals heterostructures. Nature 567, 71-75 (2019).

4. Seyler, K. L. et al. Signatures of moiré-trapped valley excitons in $\mathrm{MoSe}_{2} / \mathrm{WSe}_{2}$ heterobilayers. Nature 567, 66-70 (2019).
5. Jin, C. et al. Observation of moiré excitons in $\mathrm{WSe}_{2} / \mathrm{WS}_{2}$ heterostructure superlattices. Nature 567, 76-80 (2019).

6. Palacios-Berraquero, C. et al. Large-scale quantum-emitter arrays in atomically thin semiconductors. Nat. Commun. 8, 15093 (2017).

7. Mrejen, M., Yadgarov, L., Levanon, A. \& Suchowski, H. Transient excitonpolariton dynamics in $\mathrm{WSe}_{2}$ by ultrafast near-field imaging. Sci. Adv. $\mathbf{5}$, eaat9618 (2019)

8. Sternbach, A. J. et al. Programmable hyperbolic polaritons in van der Waals semiconductors. Science 371, 617 (2021).

9. Raja, A. et al. Coulomb engineering of the bandgap and excitons in twodimensional materials. Nat. Commun. 8, 15251 (2017).

10. Bao, W. et al. Visualizing nanoscale excitonic relaxation properties of disordered edges and grain boundaries in monolayer molybdenum disulfide. Nat. Commun. 6, 7993 (2015)

11. Park, K.-D. et al. Hybrid tip-enhanced nanospectroscopy and nanoimaging of monolayer WSe ${ }_{2}$ with local strain control. Nano Lett. 16, 2621-2627 (2016).

12. Park, K.-D., Jiang, T., Clark, G., Xu, X. \& Raschke, M. B. Radiative control of dark excitons at room temperature by nano-optical antenna-tip Purcell effect. Nat. Nanotechnol. 13, 59-64 (2018).

13. Xue, W. et al. Nano-optical imaging of monolayer $\mathrm{MoSe}_{2}-\mathrm{WSe}_{2}$ lateral heterostructure with subwavelength domains. J. Vac. Sci. Technol. A $\mathbf{3 6}$ 05G502 (2018).

14. Tang, C. et al. Quantum plasmonic hot-electron injection in lateral $\mathrm{WSe}_{2} /$ $\mathrm{MoSe}_{2}$ heterostructures. Phys. Rev. B 98, 041402 (2018).

15. He, Z. et al. Quantum plasmonic control of trions in a picocavity with monolayer $\mathrm{WS}_{2}$. Sci. Adv. 5, eaau8763 (2019).

16. Park, K.-D. et al. Tip-enhanced strong coupling spectroscopy, imaging, and control of a single quantum emitter. Sci. Adv. 5, eaav5931 (2019).

17. Lee, H. et al. Tip-enhanced photoluminescence nano-spectroscopy and nanoimaging. Nanophotonics 9, 3089-3110 (2020).

18. May, M. A. et al. Nanocavity clock spectroscopy: Resolving competing exciton dynamics in $\mathrm{WSe}_{2} / \mathrm{MoSe}_{2}$ heterobilayers. Nano Lett. 21, 522-528 (2021).

19. Darlington, T. P. et al. Imaging strain-localized excitons in nanoscale bubbles of monolayer $\mathrm{WSe}_{2}$ at room temperature. Nat. Nanotechnol. 15, 854-860 (2020). 
20. Chen, X. et al. Modern scattering-type scanning near-field optical microscopy for advanced material research. Adv. Mater. 31, 1804774 (2019).

21. Fei, Z. et al. Gate-tuning of graphene plasmons revealed by infrared nanoimaging. Nature 487, 82-85 (2012).

22. Chen, J. et al. Optical nano-imaging of gate-tunable graphene plasmons. Nature 487, 77-81 (2012).

23. Dai, S. et al. Tunable phonon polaritons in atomically thin van der Waals crystals of boron nitride. Science 343, 1125 (2014).

24. Ogawa, Y., Minami, F., Abate, Y. \& Leone, S. R. Nanometer-scale dielectric constant of Ge quantum dots using apertureless near-field scanning optical microscopy. Appl. Phys. Lett. 96, 063107 (2010).

25. Ocelic, N., Huber, A. \& Hillenbrand, R. Pseudoheterodyne detection for background-free near-field spectroscopy. Appl. Phys. Lett. 89, 101124 (2006).

26. Knoll, B. \& Keilmann, F. Enhanced dielectric contrast in scattering-type scanning near-field optical microscopy. Opt. Commun. 182, 321-328 (2000).

27. Caldwell, J. D. et al. Photonics with hexagonal boron nitride. Nat. Rev. Mater. 4, 552-567 (2019).

28. Govyadinov, A. A., Amenabar, I., Huth, F., Carney, P. S. \& Hillenbrand, R. Quantitative measurement of local infrared absorption and dielectric function with tip-enhanced near-field microscopy. J. Phys. Chem. Lett. 4, 1526-1531 (2013).

29. Glazov, M. M. et al. Exciton fine structure and spin decoherence in monolayers of transition metal dichalcogenides. Phys. Rev. B 89, 201302 (2014).

30. Scuri, G. et al. Large excitonic reflectivity of monolayer $\mathrm{MoSe}_{2}$ encapsulated in hexagonal boron nitride. Phys. Rev. Lett. 120, 037402 (2018).

31. Epstein, I. et al. Near-unity light absorption in a monolayer $\mathrm{WS}_{2}$ van der Waals heterostructure cavity. Nano Lett. 20, 3545-3552 (2020).

32. Fei, Z. et al. Infrared nanoscopy of Dirac plasmons at the graphene-SiO interface. Nano Lett. 11, 4701-4705 (2011).

33. Zhang, L. M. et al. Near-field spectroscopy of silicon dioxide thin films. Phys. Rev. B 85, 075419 (2012).

34. Palummo, M., Bernardi, M. \& Grossman, J. C. Exciton radiative lifetimes in two-dimensional transition metal dichalcogenides. Nano Lett. 15, 2794-2800 (2015).

35. Poellmann, C. et al. Resonant internal quantum transitions and femtosecond radiative decay of excitons in monolayer $\mathrm{WSe}_{2}$. Nat. Mater. 14, 889-893 (2015).

36. Robert, C. et al. Exciton radiative lifetime in transition metal dichalcogenide monolayers. Phys. Rev. B 93, 205423 (2016).

37. Moody, G. et al. Intrinsic homogeneous linewidth and broadening mechanisms of excitons in monolayer transition metal dichalcogenides. Nat. Commun. 6, 8315 (2015).

38. Epstein, I. et al. Highly confined in-plane propagating exciton-polaritons on monolayer semiconductors. 2D Mater. 7, 035031 (2020).

39. Basov, D. N., Asenjo-Garcia, A., Schuck, P. J., Zhu, X. \& Rubio, A. Polariton panorama. Nanophotonics 10, 549-577 (2021).

40. Barachati, F. et al. Interacting polariton fluids in a monolayer of tungsten disulfide. Nat. Nanotechnol. 13, 906-909 (2018).

41. Bao, W. et al. Observation of Rydberg exciton polaritons and their condensate in a perovskite cavity. Proc. Natl Acad. Sci. USA 116, 20274 (2019).

42. Wang, G. et al. In-plane propagation of light in transition metal dichalcogenide monolayers: Optical selection rules. Phys. Rev. Lett. 119 047401 (2017).

43. Zhang, X.-X. et al. Magnetic brightening and control of dark excitons in monolayer $\mathrm{WSe}_{2}$. Nat. Nanotechnol. 12, 883-888 (2017).

44. Fali, A. et al. Nanoscale spectroscopy of dielectric properties of Mica. ACS Photonics 8, 175-181 (2021).

45. Zhang, X.-X., You, Y., Zhao, S. Y. F. \& Heinz, T. F. Experimental evidence for dark excitons in monolayer $\mathrm{WSe}_{2}$. Phys. Rev. Lett. 115, 257403 (2015).

46. Jin, C. et al. Identification of spin, valley, and moiré quasi-angular momentum of interlayer excitons. Nat. Phys. 15, 1140-1144 (2019).

47. Edelberg, D. et al. Approaching the intrinsic limit in transition metal diselenides via point defect control. Nano Lett. 19, 4371-4379 (2019).

48. Ross, J. S. et al. Electrical control of neutral and charged excitons in a monolayer semiconductor. Nat. Commun. 4, 1474 (2013).

49. Chernikov, A. et al. Exciton binding energy and nonhydrogenic Rydberg series in monolayer $\mathrm{WS}_{2}$. Phys. Rev. Lett. 113, 076802 (2014)

50. Hsu, W.-T. et al. Negative circular polarization emissions from $\mathrm{WSe}_{2} / \mathrm{MoSe}_{2}$ commensurate heterobilayers. Nat. Commun. 9, 1356 (2018).

51. Nayak, P. K. et al. Probing evolution of twist-angle-dependent interlayer excitons in $\mathrm{MoSe}_{2} / \mathrm{WSe}_{2}$ van der Waals heterostructures. ACS Nano 11, 4041-4050 (2017).

52. Alexeev, E. M. et al. Resonantly hybridized excitons in moiré superlattices in van der Waals heterostructures. Nature 567, 81-86 (2019).
53. Borghardt, S. et al. Engineering of optical and electronic band gaps in transition metal dichalcogenide monolayers through external dielectric screening. Phys. Rev. Mater. 1, 054001 (2017).

54. Arora, A. et al. Excitonic resonances in thin films of $\mathrm{WSe}_{2}$ : From monolayer to bulk material. Nanoscale 7, 10421-10429 (2015).

55. Liu, K. et al. Evolution of interlayer coupling in twisted molybdenum disulfide bilayers. Nat. Commun. 5, 4966 (2014).

56. Tang, Y. et al. Simulation of Hubbard model physics in $\mathrm{WSe}_{2} / \mathrm{WS}_{2}$ moiré superlattices. Nature 579, 353-358 (2020).

57. Shimazaki, Y. et al. Strongly correlated electrons and hybrid excitons in a moiré heterostructure. Nature 580, 472-477 (2020).

58. Regan, E. C. et al. Mott and generalized Wigner crystal states in $\mathrm{WSe}_{2} / \mathrm{WS}_{2}$ moiré superlattices. Nature 579, 359-363 (2020).

59. Stern, M., Umansky, V. \& Bar-Joseph, I. Exciton liquid in coupled quantum wells. Science 343, 55 (2014).

60. Fogler, M. M., Butov, L. V. \& Novoselov, K. S. High-temperature superfluidity with indirect excitons in van der Waals heterostructures. Nat. Commun. 5 4555 (2014).

61. Li, Y. et al. Measurement of the optical dielectric function of monolayer transition-metal dichalcogenides: $\mathrm{MoS}_{2}, \mathrm{MoSe}_{2}, \mathrm{WS}_{2}, \mathrm{WSe}_{2}$. Phys. Rev. B 90 205422 (2014)

\section{Acknowledgements}

Experimental research at Columbia is supported as part of Programmable Quantum Materials, an Energy Frontier Research Center funded by the U.S. Department of Energy (DOE), Office of Science, Basic Energy Sciences (BES), under award DESC0019443. F.M gratefully acknowledges support by the Alexander von Humboldt Foundation. Authors thank Yilei Li for providing the dielectric function data of $\mathrm{WSe}_{2}$

\section{Author contributions}

S.Z. and D.N.B. designed the experiments. B.C.L., W.J.W., S.S., L.Z., Z.Y.W., E.R., N.W., C.R.D., A.N.P., X.X., X.Y.Z. and J.H. designed and fabricated the devices. X.Y.X. and P.J.S. characterized the sample twist angle. S.Z. performed the experimental measure ments with input from Y.M.S., A.J.S. and L.X. S.Z. and D.N.B analyzed the experimental data. X.Z.C., M.K.L. and F.L.R. developed the theoretical model to analyze the experimental data with input from A.J.S., A.S.M., Z.S., S.L.M., F.M., M.L. and A.J.M. S.Z. and D.N.B. co-wrote the manuscript with input from all co-authors. D.N.B. supervised the project. All authors contributed to the discussions.

\section{Competing interests}

The authors declare no competing interests.

\section{Additional information}

Supplementary information The online version contains supplementary material available at https://doi.org/10.1038/s41467-022-28117-x.

Correspondence and requests for materials should be addressed to D. N. Basov.

Peer review information Nature Communications thanks Yohannes Abate, Alexander Govyadinov, and Dmitri Voronine for their contribution to the peer review of this work. Peer reviewer reports are available.

Reprints and permission information is available at http://www.nature.com/reprints

Publisher's note Springer Nature remains neutral with regard to jurisdictional claims in published maps and institutional affiliations.

Open Access This article is licensed under a Creative Commons Attribution 4.0 International License, which permits use, sharing, adaptation, distribution and reproduction in any medium or format, as long as you give appropriate credit to the original author(s) and the source, provide a link to the Creative Commons license, and indicate if changes were made. The images or other third party material in this article are included in the article's Creative Commons license, unless indicated otherwise in a credit line to the material. If material is not included in the article's Creative Commons license and your intended use is not permitted by statutory regulation or exceeds the permitted use, you will need to obtain permission directly from the copyright holder. To view a copy of this license, visit http://creativecommons.org/ licenses/by/4.0/

(c) The Author(s) 2022 